\title{
Traditional and Non-Traditional Risk Factors Involved with Endothelial Dysfunction and Arterial Stiffness in Autoimmune Rheumatic Diseases
}

\author{
Rafael Giovani Misse, Isabela Bruna Pires Borges, Samuel Katsuyuki Shinjo* \\ Division of Rheumatology, Faculdade de Medicina FMUSP, Universidade de Sao Paulo, Sao Paulo, Brazil \\ Email: *samuel.shinjo@gmail.com
}

How to cite this paper: Misse, R.G., Borges, I.B.P. and Shinjo, S.K. (2019) Traditional and Non-Traditional Risk Factors Involved with Endothelial Dysfunction and Arterial Stiffness in Autoimmune Rheumatic Diseases. Open Journal of Rheumatology and Autoimmune Diseases, 9, 85-100. https://doi.org/10.4236/ojra.2019.93008

Received: April 17, 2019

Accepted: August 9, 2019

Published: August 12, 2019

Copyright $\odot 2019$ by author(s) and Scientific Research Publishing Inc. This work is licensed under the Creative Commons Attribution International License (CC BY 4.0).

http://creativecommons.org/licenses/by/4.0/

\begin{abstract}
Autoimmune rheumatic diseases (ARDs) have been closely associated with accelerated plaque progression and the development of atherosclerosis, which lead to high morbidity and mortality rates for cardiovascular diseases. Endothelial dysfunction and arterial stiffness are greatly evidenced in several studies in the early phase of atherosclerosis. In ARDs, endothelial dysfunction and arterial stiffness are related to traditional and non-traditional risk factors. To date, no studies have clearly analyzed the main parameter involved in endothelial dysfunction and arterial stiffness. In this context, the present narrative review's purpose was to describe the main factor in endothelial dysfunction and arterial stiffness in different ARDs. Endothelial dysfunction and arterial stiffness are related to traditional risk factors (i.e., hypertension, diabetes, dyslipidemia, metabolic syndrome, sedentary behavior) and non-traditional risk factors (linked to the immune mechanisms involved in these diseases). Moreover, in the present study, these associations were systemically analyzed in ankylosing spondylitis, antiphospholipid syndrome, rheumatoid arthritis, psoriatic arthritis, systemic autoimmune myopathies, systemic lupus erythematosus and systemic sclerosis. The present review shows that the relationship of traditional risk factors and non-traditional risk factors related to ARDs works in the worsening of function and structural properties of arterial vessels, leading to high cardiovascular morbidity and mortality.
\end{abstract}

\section{Keywords}

Arterial Stiffness, Atherosclerosis, Autoimmune Endothelial Dysfunction, Rheumatic Diseases 


\section{Introduction}

Autoimmune rheumatic diseases (ARDs) are a heterogeneous group of disorders commonly characterized by systemic and chronic inflammatory processes [1]. A high frequency of morbidity and mortality related to cardiovascular diseases (CVD), particularly myocardium infarction, stroke and congestive heart failure has been described in several ARDs [1] [2].

Cardiovascular morbidity and mortality are strongly associated with accelerated atherosclerotic processes, which are preceded by endothelial dysfunction and arterial stiffness [3]. Therefore, the early assessment of endothelial function and arterial stiffness is a useful predictor of CVD risk. It may be accessed via flow-mediated dilatation (FMD) in the former case, and pulse wave velocity (PWV) in the latter [4].

Of note, endothelial dysfunction and increased arterial stiffness have been described in many ARDs, such as ankylosing spondylitis (AS), antiphospholipid syndrome (APS), rheumatoid arthritis (RA), psoriatic arthritis (PsA), systemic autoimmune myopathies (SAM), systemic lupus erythematosus (SLE) and systemic sclerosis (SSc) [4]-[9].

Impaired endothelial and arterial functions in ARDs may be explained by traditional risk factors (TRFs) for CVDs, such as systemic arterial hypertension, diabetes mellitus, tobacco habit, obesity and sedentary behavior [10]. In addition, recent data have supported the participation of non-TRFs in CVD, such as cellular and humoral immunity [6] (Figure 1).

No studies have clearly analyzed these ARD factors. In fact, the knowledge of these factors and their impact may contribute to a therapy approach even in the early phase of atherosclerosis [8] [9]. In this context, the present study's purposes were to describe the main parameter involved in endothelial dysfunction and arterial stiffness in ARDs.

\subsection{Literature Review}

We conducted literature searches on electronic databases (Medline [PubMed]). The selection of the descriptors was carried out from June 2018 to November 2018, according to the Medical Subject Headings, following key-words in English: "ankylosing spondylitis", "antiphospholipid syndrome", "arterial stiffness", "autoimmune diseases", "dermatomyositis", "endothelial dysfunction", "idiopathic inflammatory myopathies", "myositis", "polymyositis", "psoriatic arthritis", "rheumatic diseases", "spondyloarthritis", "systemic autoimmune diseases", "systemic autoimmune myopathies", "systemic lupus erythematosus", "systemic sclerosis". The keywords were combined with the Boolean operators "AND" and "OR" and adapted for each database as required. In addition, the reference list of each retrieved article was reviewed manually by two reviewers and in case of disagreement a third reviewer was called. The following inclusion criteria were adopted: no chronological limit, original articles, reviews, controlled and randomized clinical trials. 


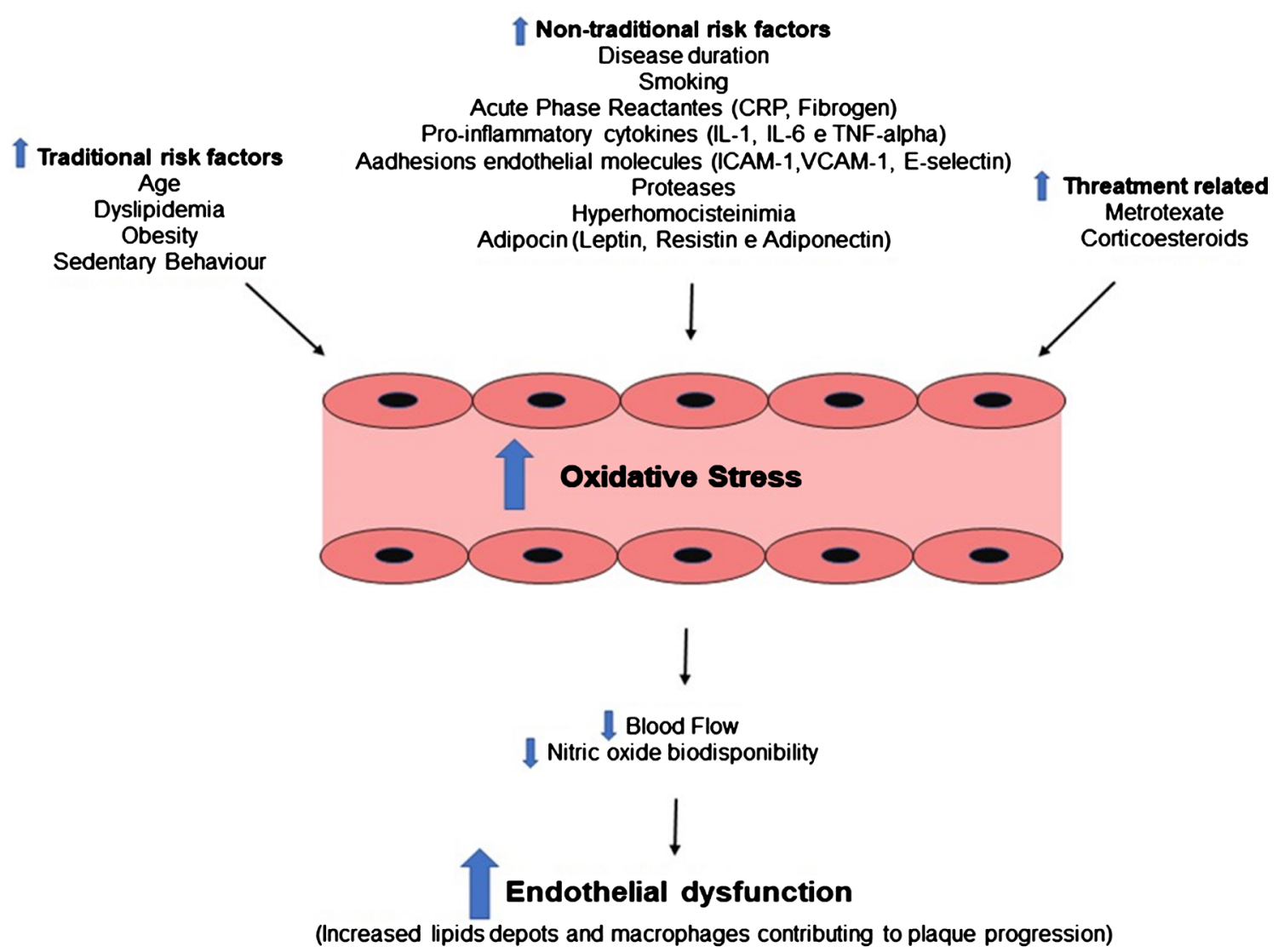

Figure 1. The traditional and non-traditional risk factors to the endothelial dysfunction.

\subsubsection{Ankylosing Spondylitis}

The literature contains less information about the assessment of endothelial function in AS [11] [12]. Several studies have shown arterial stiffness in patient's large arteries [11] [12]. To our knowledge, the studies have not demonstrated an increase in the common (carotid intima-media thickness) cIMT [11]. Moreover, no studies have evaluated the PWV. Regarding FMD, an important impairment was shown [12]. The possible mechanism involved was related to impairments in the anti-atherogenic factors linked to high density protein [11] [12], but limited studies have elucidated the potential mechanism involved the vascular dysfunction of these patients.

\subsubsection{Antiphospholipid Syndrome}

APS has often been linked with arterial and thromboembolic events [6]. Unlike in RA and SLE, the TRFs in APS are common among those in the general population, suggesting that TRFs are less important [6].

The non-TRFs in APS are related to auto-antibodies such as anti-oxidized low-density lipoproteins (anti-oxLDL), anti-heat shock protein (HSP), anti-cardiolipin and anti-beta-2-glycoprotein I (anti- $\beta 2$ GPI) [13] [14]. These autoantibodies can influence the subendothelial lipids retention, leading to accelerated atherosclerosis and other CVDs, such as cerebrovascular events and peripheral artery disease [13]. 
The presence of anti-oxLDL and $\beta$ 2GPI complexes and anti-heat shock protein antibodies are described in atherosclerosis [13]. $\beta$ 2GPI is expressed in adjacent CD4+ T cell infiltrations. During the progression of atherosclerosis, macrophages and endothelial cells bind to $\beta$ 2GPI [13] [14]. The anti-oxLDL and anti- $\beta$ 2GPI have the strongest association with the common cIMT and endothelial dysfunction [13] [14]. In the same way, the relationship between anti-cardiolipin and increased common cIMT was observed [13]. These autoantibodies correlate with the frequency of CVD [13]. Similar findings have been founded with the IgA isotype, which was linked to cardiovascular events (stable and unstable angina) [6]. Recently, one study revealed that the activation of the mammalian target of the rapamycin complex rather than the phosphatidyl-inositol 3 kinase pathway stimulates the intima, promoting chronic vascular disease. However, this association remains unclear, requiring more studies [14]. Moreover, the vascular structure and function in APS-impaired FMD and increased common cIMT [15].

\subsubsection{Rheumatoid Arthritis}

The most frequently described TRFs for CVD in RA are smoking, sedentary behavior and dyslipidemia [1] [16], whereas mainly non-TRFs are factors in this disease's chronic and systemic inflammatory process [17] [18], as shown in Table 1.

Smoking is an independent risk factor for RA [1]. A positive relationship exists between smoking and worse prognoses and disease severity [1] [19]. The probable mechanism is related to the increase of anti-citrullinated protein/peptide antibodies, leading to increased disease severity and a worse prognosis [1] [19].

These factors are related to other TRFs, such as hypertension, dyslipidemia, obesity and sedentary behavior, which contribute to worse prognoses [6] [16] [20] [21]. In the same way, joint deformities can lead to a decrease in muscle function and strength, contributing to increased sedentary behavior [24]. Recently, one study demonstrated that the impairment of microvascular endothelium-dependent vasodilation was correlated with increased sedentary behavior [16].

Table 1. Main risk factors for cardiovascular diseases involved in possible endothelium dysfunction and/or arterial stiffness in rheumatoid arthritis.

\begin{tabular}{|c|c|c|c|}
\hline \multicolumn{3}{|c|}{ Main risk factors for cardiovascular diseases Relationship } & References \\
\hline \multirow[t]{5}{*}{ Traditional } & Smoking & & \multirow{3}{*}{$\begin{array}{lll}{[1]} & {[6]} & {[16]} \\
{[17]} & {[18]} & {[20]} \\
{[21]} & {[22]} & \end{array}$} \\
\hline & Ohesity & & \\
\hline & Ooesity & Controversial: protective effect, deleterious effect on the disease's severity. & \\
\hline & Sedentary behavior & Correlates to the impairment of microvascular endothelium-dependent vasodilation. & \\
\hline & Dyslipidemia & & \\
\hline \multirow[t]{4}{*}{ Non-traditional } & Disease' chronic and & Involving the presence of pro-inflammatory cytokines, chemokines, endothelial & \multirow{4}{*}{$\begin{array}{l}{[17][23][25]} \\
{[26][27][28]}\end{array}$} \\
\hline & systemic inflammatory & adhesion molecules, vascular endothelial growth factors, C-reactive protein, and & \\
\hline & process & platelet-derived growth factors. & \\
\hline & & Contributes to insulin resistance, dyslipidemia, obesity, hiperhomocysteinemia. & \\
\hline
\end{tabular}


Another important TRF is obesity [23] [24]. Considered a paradox, obesity in some studies exerts a potential protective effect through adiponectin, resistin and adipocytokines, but the obesity seems to have a deleterious effect on the disease's severity [23] [24]. However, researchers have reached a consensus on endothelial dysfunction and obesity [24].

Obesity is linked to dyslipidemia in the general population and in RA patients [23] [25]. The prevalence of expressive dyslipidemia in RA is related to abnormalities in lipid profiles [25]. One cross-sectional study identified genes (TRAF1/C5, STAT4 and HLA-DRB1-SE) susceptibility linked to lipid metabolism in RA [26].

The non-TRFs involved in AR mainly feature chronic systemic inflammation, involving the presence of pro-inflammatory cytokines [i.e., tumor necrosis factor (TNF), interleukin (IL)-1, IL-6 and interferon (IFN) @], chemokines, endothelial adhesion molecules, vascular endothelial growth factors (VEGF), C-reactive protein, and platelet-derived growth factors (PDGF) [17] [27].

In relation to the pro-inflammatory cytokines in RA, the involvement of cytokines such as TNF, IL-1 and IL-6 has been shown [9]. High levels of IL-6 and TNF have been positively correlated with some TRFs, insulin resistance, dyslipidemia, and obesity, respectively [9].

It has also been shown that IL-1/TNF can cause alterations in serum concentrations of homocysteine, leading to the production of asymmetric dimethylarginine, which negatively affects the regulating expression of nitric oxide synthase (NOS) [17].

Homocysteinemia seems to be a non-TRF in RA and is linked to folate and B12 metabolism [28]. A deficiency in B12 or folate metabolism can lead to homocysteinemia, causing endothelial damage and contributing to smooth muscle cell proliferation [28].

IL-1/TNF is also related to the expression of homocysteine, which is responsible for regulating paraoxonase, which is closely linked with increased cardiovascular risk and mortality [28]. Moreover, the relationship between these cytokines with higher serum concentrations of endothelial adhesion molecules in RA was shown [27].

From a clinical perspective, decreased endothelial function in RA led to an association between TNF, IL-1 and IL- 6 with decreased FMD, similar to disease parameters, which were linked to a decrease in FMD [29]. In agreement with these findings, current evidence demonstrates an important reduction in vascular endothelium-dependent vasodilatation, which is correlated with parameters of clinical disease activity [30]. Caraba et al. [31] reported similar findings, demonstrating that disease activity is positively correlated with insulin resistance and endothelial dysfunction [31].

These parameters were identified with disease activity, and after one year, the reduction in disease activity improved the endothelial function, suggesting that chronic inflammation is potentially responsible for impairments in endothelial 
function and arterial stiffness [32] [33].

According to the literature, a significant reduction of FMD and cIMT was observed in 87 patients, as well as the irregular expansion of CD4+ and CD28 lymphocytes, similar to patients with CVDs [34].

The emergent association was seen in metalloproteinase and endothelial dysfunction [35]. In RA, metalloproteinases are linked to joint destruction and chronic systemic inflammation. Moreover, metalloproteinases are related to plaque destabilization [35] [36].

In contrast, some studies showed that the prevalence of TRFs is the main determinant of endothelial dysfunction and arterial stiffness in RA patients [37] [38]. These findings are found in a cross-sectional and longitudinal study, which evaluated the connection between inflammatory parameters, TRFs and macro-microvascular function [37]. In the same sense, it was evidenced by the same group in a prospective six-year study [38].

Sandoo et al. observed an increase in the common cIMT. This impairment was strongly bound with CVD in patients without a history of vascular disease [37].

Giles et al. [39] in a study with mixed ARDs involving 101 patients (including patients with RA) observed the presence of arterial stiffness by angiography, the PWV and FMD [39]. The PWV was correlated with reduced FMD and increased cIMT. According to previous findings, FMD is a strong predictor of atherosclerosis [39].

In agreement with these studies, a recent study that enrolled 5638 patients showed that $70 \%$ of cardiovascular events were attributed to TRF, and $40 \%$ were attributed to RA [40]. Finally, smoking and systemic arterial hypertension have a greater impact on global CVD risk factors [40].

In $\mathrm{RA}$, the risk factors are associated with the disease, and the traditional risk factors for CVDs together contribute to a significant increase in morbidity and mortality in RA. In addition, chronic inflammatory response results in an important progression of traditional risk factors, ultimately leading to decreased FMD. The potential determinants of these impairments are disease activity or remission, chronic systemic inflammation and traditional risk factors [2] [9] [19] [37] [38].

All of these factors seem to contribute to endothelial dysfunction and arterial stiffness in RA [19] [41]. In RA, endothelial dysfunction can occur in the large vessels (such as the conduit arteries) and the small vessels of the microvasculature, which supply oxygen and nutrients to the tissue and control inflammation, repair and fluid exchange with the surrounding tissues [19]. Moreover, patients with RA show a higher prevalence of arterial stiffness. This alteration correlates with the severity of the inflammatory status and is present even in the disease's early stage [41].

\subsubsection{Psoriatic Arthritis}

The association between CVDs and PsA was recently described. As with RA and SLE, the presence of TRFs contributes to elevated cardiovascular risk, in the 
same way as non-TRFs [42] [43]. TRFs are characterized mainly by hypertriglyceridemia, obesity and smoking [42] [44].

The assessment of subclinical atherosclerosis has been discussed in several studies [43] [44]. The majority agrees that PsA is associated with a higher common cIMT and PWV, as well as decreased FMD [43] [44]. These parameters have been correlated with disease duration and age, as in RA findings [42] [43].

The common cIMT has been strongly correlated with the severity of PsA, age and disease duration [45]. These findings were similar in a large cohort of patients, showing that psoriasis represents independent CVD risk factors [46]. As observed in the study that Balci et al. [47] conducted disease duration is associated with increased cIMT [47]. Regarding the PWV, it has been shown that arterial stiffness is related to age and disease duration [42] [46]. These findings were similar after a multivariate regression analysis using the age, gender, smoking, hypertension, and body mass index dependent variables [42] [45].

A functional assessment by FMD revealed a considerable reduction in some studies [42] [45] [46]. However, these observations did not correlate disease duration with the reduction of FMD [42] [45] [46] [47]. In contrast, some studies did not show statistically significant differences between the groups [48]. Further studies are needed to elucidate the functional characteristics of the arterial arteries of patients with PsA.

\subsubsection{Systemic Autoimmune Myopathies}

To date, only one study evaluated endothelial function and arterial stiffness in patients with SAM [5]. This study demonstrated the presence of arterial stiffness measured by the common cIMT, which was not statistically significant among 38 health controls and 21 patients with dermatomyositis and polymyositis. Regarding endothelial function, a significant reduction of endothelium-dependent vasodilation was observed, suggesting that it could be related to chronic inflammation in the skeletal muscle [5]. However, regarding limitations, this study did not assess clinical, laboratory, or overall CVDs and their risk factors. Therefore, endothelial function and arterial stiffness in systemic autoimmune myopathies remain unclear. More studies are required to elucidate vessel behavior in myopathies and vice versa.

\subsubsection{Systemic Lupus Erythematosus}

Analogous to other ARDs, SLE is clinically characterized by a high incidence of CVDs [49] [50]. SLE often affects women without previous exposure to atherosclerosis [51]. However, as in other ARDs, a high frequency of CVDs after diagnosis has been observed [49] [50], as shown in Table 2.

In SLE, CVD risk is thought to increase as a result of the complex interaction between TRFs: sedentary behavior, dyslipidemia, hypertension, metabolic syndrome and insulin resistance [2] [6].

Immunity deregulation occurs due to disease activity, including the synergic effect of cytokines, chemokines, adipokines, proteases, autoantibodies, adhesion 
Table 2. Main risk factors for cardiovascular diseases involved in possible endothelium dysfunction and/or arterial stiffness in systemic lupus erythematosus.

\begin{tabular}{|c|c|c|c|}
\hline \multicolumn{2}{|c|}{ Main risk factors for cardiovascular diseases } & \multirow{2}{*}{$\begin{array}{l}\text { Relationship } \\
\text { Worse prognoses and disease severity. }\end{array}$} & \multirow{3}{*}{$\begin{array}{l}\text { References } \\
{[2][6][20]} \\
{[51][52]}\end{array}$} \\
\hline \multirow{8}{*}{ Traditional } & Smoking & & \\
\hline & Obesity & Relation to dyslipidemia. & \\
\hline & & Controversial: protective effect, deleterious effect on the disease's severity. & \\
\hline & Sedentary behavior & Correlates to the impairment of microvascular endothelium-dependent vasodilation. & \\
\hline & Hypertension & & \\
\hline & Insulin resistance & Chronic use of glucocorticoids. & \\
\hline & Metabolic syndrome & Chronic use of glucocorticoids. & \\
\hline & Dyslipidemia & Chronic use of glucocorticoids. & \\
\hline \multirow{3}{*}{ Non-traditional } & Disease' activity & $\begin{array}{l}\text { Immunity deregulation occurs due to disease activity, including the synergic effect of } \\
\text { cytokines, chemokines, adipokines, proteases, autoantibodies, adhesion receptors, } \\
\text { oxidative stress and a plethora of intracellular signaling moleculesa. }\end{array}$ & $\begin{array}{l}{[53][54][55]} \\
{[56]}\end{array}$ \\
\hline & Autoantibodies & $\begin{array}{l}\text { Antiphospholipid antibodies, anti-oxidized palmitoyl-arachidonyl-phosphocholine, } \\
\text { antibodies to } 65 \text { and } 70 \mathrm{kDa} \text { heat shock proteins (anti-hsp } 65 \text { and -hsp70), anti-HDL, } \\
\text { anti-apolipoprotein A-I and anti-lipoprotein lipase. The release of high numbers of } \\
\text { these autoantibodies may increase oxidative stress, leading to endothelial cell damage } \\
\text { and apoptosis and helping accelerate the atherosclerotic process. }\end{array}$ & \\
\hline & Cytokines & $\begin{array}{l}\text { Pro-inflammatory mechanism involved is increased by C-reactive protein, } \\
\text { adipocytokines and proteases. }\end{array}$ & \\
\hline
\end{tabular}

receptors, oxidative stress and a plethora of intracellular signaling molecules. These factors together could contribute to the impairment of endothelial function and increased arterial stiffness [20].

Among TRFs, dyslipidemia in SLE has been associated with the chronic use of glucocorticoids [51]. These parameters isolated or combined, corroborate changes in serum high density lipoprotein cholesterol levels and elevated levels of very-low-density lipoprotein cholesterol and triglycerides [51]. It has also been shown that the presence of immunocomplexes may influence endothelium's and macrophages' ability to catalyze and hydrolyze cholesterol [51].

A high frequency of metabolic syndrome has been observed among patients with SLE [20] [52]. The high frequency of metabolic syndrome has been consistently connected to ethnicity and depression [20] [52].

The non-TRFs involved in SLE mainly include by antiphospholipid antibodies (anticardiolipin and anti- $\beta 2 \mathrm{GPI}$ ), anti-oxLDL, anti-oxidized palmitoyl-arachidonylphosphocholine, antibodies to 65 and $70 \mathrm{kDa} H S P$, anti-high density lipoprotein cholesterol, anti-apolipoprotein A-I and anti-lipoprotein lipase [6]. These antibodies have been linked to the thermogenic process [53] [54]. The release of high numbers of these autoantibodies may increase oxidative stress, thus leading to endothelial cell damage and apoptosis, as well as helping to accelerate the atherosclerotic process [53] [54].

The pro-inflammatory mechanism involved is increased C-reactive protein, adipocytokines and proteases [55]. Particularly, these pro-inflammatory me- 
chanisms influence endothelial permeability and homeostasis, thus leading to further plaque destabilization [55].

C-reactive protein is a pro-atherogenic independent factor that leads to the complementary activation of chemokine and its release into the endothelium contributing to oxLDL and macrophage uptake [56]. The increased levels of oxLDL serum correlate with increased common cIMT, suggesting a strong relationship between these endothelial dysfunction and atherosclerosis factors [57]. Adipocytokines and proteases in SLE are mainly involved with endothelial injury, plaque destabilization and progression [53].

Regarding the evaluation of endothelial function and arterial stiffness in SLE, several studies have investigated vascular behavior and characteristic [2] [6].

Soltesz et al. [58] showed vascular abnormalities through the assessment of cIMT, FMD and nitroglycerine mediated dilatation (NMD) in 61 patients with systemic lupus erythematosus with to 26 matched controls. FMD was correlated with arterial hypertension and aging [58]. Similar findings were observed in arterial stiffness evaluated by the PWV and BaPWV [58] [59].

However, unlike in RA, in SLE, the decreased FMD and the increased PWV and common cIMT in SLE correlate strongly with the inflammatory mechanism, which can explain the damage in multiple organs [58] [60].

\subsubsection{Systemic Sclerosis}

SSc has been reported with inflammatory, vascular and fibrotic events. The mechanisms underlying these factors are mainly associated with macrovascular and microvascular impairments [8] [61].

The microvascular impairments feature histopathological abnormalities in the skin (Raynaud's phenomenon) that influence several molecular and cellular mechanisms [61]. The macrovascular damage is characterized by lumen abnormalities, leading to short-term endothelial injury and apoptosis [62] [63].

The factors involved in macro and microvascular deterioration cannot be explained by TRFs. Several studies have not shown a correlation with the clinical evaluation of cardiovascular risk. In contrast, several cellular and molecular mechanisms have been identified and correlated with the variated clinical evaluation of CVD risk factors [61] [64].

Regarding the relationship with TRFs and SSc, Szamosi et al. [65] demonstrated a positive correlation with elevated homocysteine levels and macrovascular diseases in 133 patients with SSc systemic and 21 SSc with diffuse manifestations. In the same study, the authors did not find a relationship between macrovascular disease and TRFs [65]. Other studies strongly support these findings [65] [66].

The cellular and molecular mechanisms are extensively interrelated with the regulation of pro-angiogenic and anti-angiogenic factors, autoantibodies and defective endothelial progenitor cells [19] [63]. The pro-angiogenic factors in SSc are VEGF and their receptors VEGFR, VEGFR-2 and VEGFR-3, PDGF, PDGF-BB, fibroblast growth factor ${ }^{\circledR}$, angiopoietin-1 and -2 [19] [63] [67]. 
The anti-angiogenic factors are metalloproteinase 12 and recently VEGF165b antiangiogenic isoform, which correlate with substantial architectural loss and damage in SSc [19].

The increased architectural loss and damage in SSc were explained by a significant increase in specific anti-endothelial antibodies leading to expressive increases in reactive oxygen species' production and oxidative stress [63] [68].

In the same way, the high expression of adhesion cell molecules was shown to increase in hypoxia [63] [68] [69] and was related to the activation of hypoxia inducible factor-1 [8]. In summary, these factors together cause a deleterious effect on micro and microvessels such as defective angiogenesis, hypoxia and defective perfusion [68] [69].

Regarding the assessment of vascular structures and functions, the relationship between FMD and increased serum endothelin-1 was deemed a positive one. It was recently reported that increased common cIMT can be preceded by impaired FMD [2] [68].

Soltesz et al. [58] observed increased common cIMT and reduced FMD, but FMD was preserved in 29 patients compared with healthy subjects. This study showed a positive relationship among age, disease duration and common cIMT. Similar findings were seen in the PWV [58].

\section{Conclusion}

The present review shows that the relationship of traditional risk factors and non-traditional risk factors is related to ARDs works in the worsening of function and structural properties of arterial vessels, leading to a high cardiovascular morbidity and mortality.

\section{Funding}

Fundação de Amparo à Pesquisa do Estado de São Paulo (FAPESP) [\#2016/23574-0] to R.G.M; FAPESP [\#2016/20371-1] to I.B.P.B; FAPESP [\#2017/13109-1] to S.K.S; Faculdade de Medicina da Universidade de Sao Paulo to S.K.S.

\section{Conflicts of Interest}

The authors declare no conflicts of interest regarding the publication of this paper.

\section{References}

[1] Nurmohamed, M.T., Heslinga, M. and Kitas, G.D. (2015) Cardiovascular Comorbidity in Rheumatic Diseases. Nature Reviews Rheumatology, 11, 693-704. https://doi.org/10.1038/nrrheum.2015.112

[2] Soltész, P., Kerekes, G., Dér, H., Szücs, G., Szántó, S., Kiss, E., et al. (2011) Comparative Assessment of Vascular Function in Autoimmune Rheumatic Diseases: Considerations of Prevention and Treatment. Autoimmunity Reviews, 10, 416-425. https://doi.org/10.1016/j.autrev.2011.01.004

[3] Sherer, Y. and Shoenfeld, Y. (2006) Mechanisms of Disease: Atherosclerosis in Au- 
toimmune Diseases. Nature Clinical Practice Rheumatology, 2, 99-106. https://doi.org/10.1038/ncprheum0092

[4] Kerekes, G., Soltész, P., Nurmohamed, M.T., Gonzalez-Gay, M.A., Turiel, M., Végh, E., et al. (2012) Validated Methods for Assessment of Subclinical Atherosclerosis in Rheumatology. Nature Reviews Rheumatology, 8, 224-234.

https://doi.org/10.1038/nrrheum.2012.16

[5] Vincze, M., Dér, H., Kerekes, G., Szodoray, P., Zeher, M., Dankó, K., et al. (2014) Decreased Flow-Mediated Dilatation with Increased Arterial Stiffness and Thickness as Early Signs of Atherosclerosis in Polymyositis and Dermatomyositis Patients. Clinical Rheumatology, 33, 1635-1641.

https://doi.org/10.1007/s10067-014-2561-y

[6] Murdaca, G., Colombo, B.M., Cagnati, P., Gulli, R., Spanò, F. and Puppo, F. (2012) Endothelial Dysfunction in Rheumatic Autoimmune Diseases. Atherosclerosis, 224, 309-317. https://doi.org/10.1016/j.atherosclerosis.2012.05.013

[7] Turesson, C., Jacobsson, L., Ryden Ahlgren, A., Sturfelt, G., Wollmer, P. and Lanne, T. (2005) Increased Stiffness of the Abdominal Aorta in Women with Rheumatoid Arthritis. Rheumatology (Oxford), 44, 896-901. https://doi.org/10.1093/rheumatology/keh607

[8] Bartoli, F., Blagojevic, J., Bacci, M., Fiori, G., Tempestini, A., Conforti, M.L., et al. (2007) Flow-Mediated Vasodilation and Carotid Intima-Media Thickness in Systemic Sclerosis. Annals of the New York Academy of Sciences, 1108, 283-290. https://doi.org/10.1196/annals.1422.030

[9] Yang, X., Chang, Y. and Wei, W. (2016) Endothelial Dysfunction and Inflammation: Immunity in Rheumatoid Arthritis. Mediators of Inflammation, 2016, Article ID: 6813016. https://doi.org/10.1155/2016/6813016

[10] Siti, H.N., Kamisah, Y. and Kamsiah, J. (2015) The Role of Oxidative Stress, Antioxidants and Vascular Inflammation in Cardiovascular Disease: A Review. Vascular Pharmacology, 71, 40-56. https://doi.org/10.1016/j.vph.2015.03.005

[11] Verma, I., Syngle, A., Krishan, P. and Garg, N. (2017) Endothelial Progenitor Cells as a Marker of Endothelial Dysfunction and Atherosclerosis in Ankylosing Spondylitis: A Cross-Sectional Study. International Journal of Angiology, 26, 36-42. https://doi.org/10.1055/s-0036-1593445

[12] Sari, I., Okan, T., Akar, S., Cece, H., Altay, C., Secil, M., et al. (2006) Impaired Endothelial Function in Patients with Ankylosing Spondylitis. Rheumatology (OXford), 45, 283-286. https://doi.org/10.1093/rheumatology/kei145

[13] Lopez, L.R., Dier, K.J., Lopez, D., Merrill, J.T. and Fink, C.A. (2004) Anti-Beta 2-Glycoprotein I and Antiphosphatidylserine Antibodies Are Predictors of Arterial Thrombosis in Patients with Antiphospholipid Syndrome. American Journal of Clinical Pathology, 121, 142-149. https://doi.org/10.1309/YVQ6PX76XMYM3J29

[14] Corban, M.T., Duarte-Garcia, A., McBane, R.D., Matteson, E.L., Lerman, L.O. and Lerman, A. (2017) Antiphospholipid Syndrome: Role of Vascular Endothelial Cells and Implications for Risk Stratification and Targeted Therapeutics. Journal of the American College of Cardiology, 69, 2317-2330. https://doi.org/10.1016/j.jacc.2017.02.058

[15] Lima, D.S., Sato, E.I., Lima, V.C., Miranda, F. and Hatta, F.H. (2002) Brachial Endothelial Function Is Impaired in Patients with Systemic Lupus Erythematosus. The Journal of Rheumatology, 29, 292-297.

[16] Fenton, S.A.M., Sandoo, A., Metsios, G.S., Duda, J.L., Kitas, G.D. and Veldhuijzen van Zanten, J.J.C.S. (2018) Sitting Time Is Negatively Related to Microvascular En- 
dothelium-Dependent Function in Rheumatoid Arthritis. Microvascular Research, 117, 57-60. https://doi.org/10.1016/j.mvr.2018.01.005

[17] Garg, N., Syngle, A. and Krishan, P. (2017) Nitric Oxide: Link between Inflammation and Endothelial Dysfunction in Rheumatoid Arthritis. International Journal of Angiology, 26, 165-169. https://doi.org/10.1055/s-0036-1597577

[18] Totoson, P., Maguin-Gaté, K., Nappey, M., Wendling, D. and Demougeot, C. (2016) Endothelial Dysfunction in Rheumatoid Arthritis: Mechanistic Insights and Correlation with Circulating Markers of Systemic Inflammation. PLoS ONE, 11, e0146744. https://doi.org/10.1371/journal.pone.0146744

[19] Bordy, R., Totoson, P., Prati, C., Marie, C., Wendling, D. and Demougeot, C. (2018) Microvascular Endothelial Dysfunction in Rheumatoid Arthritis. Nature Reviews Rheumatology, 14, 404-420. https://doi.org/10.1038/s41584-018-0022-8

[20] Legge, A., Blanchard, C. and Hanly, J.G. (2017) Physical Activity and Sedentary Behavior in Patients with Systemic Lupus Erythematosus and Rheumatoid Arthritis. Open Access Rheumatology, 9, 191-200. https://doi.org/10.2147/OARRR.S148376

[21] Pinto, A.J., Roschel, H., de Sá Pinto, A.L., Lima, F.R., Pereira, R.M.R., Silva, C.A., et al. (2017) Physical Inactivity and Sedentary Behavior: Overlooked Risk Factors in Autoimmune Rheumatic Diseases? Autoimmunity Reviews, 16, 667-674.

https://doi.org/10.1016/j.autrev.2017.05.001

[22] Fenton, S.A.M., Veldhuijzen van Zanten, J., Duda, J.L., Metsios, G.S. and Kitas, G.D. (2018) Sedentary Behaviour in Rheumatoid Arthritis: Definition, Measurement and Implications for Health. Rheumatology (Oxford), 57, 213-226.

https://doi.org/10.1093/rheumatology/kex053

[23] Stavropoulos-Kalinoglou, A., Metsios, G.S., Koutedakis, Y. and Kitas, G.D. (2011) Obesity in Rheumatoid Arthritis. Rheumatology (Oxford), 50, 450-462. https://doi.org/10.1093/rheumatology/keq266

[24] Williams, I.L., Chowienczyk, P.J., Wheatcroft, S.B., Patel, A.G., Sherwood, R.A., Momin, A., et al. (2005) Endothelial Function and Weight Loss in Obese Humans. Obesity Surgery, 15, 1055-1060. https://doi.org/10.1381/0960892054621134

[25] Erum, U., Ahsan, T. and Khowaja, D. (2017) Lipid Abnormalities in Patients with Rheumatoid Arthritis. Pakistan Journal of Medical Sciences, 33, 227-230. https://doi.org/10.12669/pjms.331.11699

[26] Toms, T.E., Panoulas, V.F., Smith, J.P., Douglas, K.M., Metsios, G.S., Stavropoulos-Kalinoglou, A., et al. (2011) Rheumatoid Arthritis Susceptibility Genes Associate with Lipid Levels in Patients with Rheumatoid Arthritis. Annals of the Rheumatic Diseases, 70, 1025-1032. https://doi.org/10.1136/ard.2010.144634

[27] Foster, W., Carruthers, D., Lip, G.Y. and Blann, A.D. (2010) Inflammatory Cytokines, Endothelial Markers and Adhesion Molecules in Rheumatoid Arthritis: Effect of Intensive Anti-Inflammatory Treatment. Journal of Thrombosis and Thrombolysis, 29, 437-442. https://doi.org/10.1007/s11239-009-0370-y

[28] Pushpakumar, S., Kundu, S. and Sen, U. (2014) Endothelial Dysfunction: The Link between Homocysteine and Hydrogen Sulfide. Current Medicinal Chemistry, 21, 3662-3672. https://doi.org/10.2174/0929867321666140706142335

[29] de Groot, L., Jager, N.A., Westra, J., Smit, A.J., Kallenberg, C.G., Posthumus, M.D., et al. (2015) Does Reduction of Disease Activity Improve Early Markers of Cardiovascular Disease in Newly Diagnosed Rheumatoid Arthritis Patients? Rheumatology (Oxford), 54, 1257-1261. https://doi.org/10.1093/rheumatology/keu459

[30] Hinkema, H.J., Nienhuis, H.L., de Groot, L., Smit, A.J., van Roon, A.M., Bijl, M., et al. (2011) Is Small Artery Elasticity Decreased Prior to Intima-Media Thickening in 
Patients with Longstanding Rheumatoid Arthritis? The Journal of Rheumatology, 38, 2133-2140. https://doi.org/10.3899/jrheum.100989

[31] Caraba, A., Crisan, V., Romosan, I., Mozos, I. and Murariu, M. (2017) Vitamin D Status, Disease Activity, and Endothelial Dysfunction in Early Rheumatoid Arthritis Patients. Disease Markers, 2017, Article ID: 5241012. https://doi.org/10.1155/2017/5241012

[32] Giles, J.T., Danielides, S., Szklo, M., Post, W.S., Blumenthal, R.S., Petri, M., et al. (2015) Insulin Resistance in Rheumatoid Arthritis: Disease-Related Indicators and Associations with the Presence and Progression of Subclinical Atherosclerosis. Arthritis \& Rheumatology, 67, 626-636. https://doi.org/10.1002/art.38986

[33] Van Doornum, S., McColl, G., Jenkins, A., Green, D.J. and Wicks, I.P. (2003) Screening for Atherosclerosis in Patients with Rheumatoid Arthritis: Comparison of Two in Vivo Tests of Vascular Function. Arthritis \& Rheumatology, 48, 72-80. https://doi.org/10.1002/art.10735

[34] Gerli, R., Schillaci, G., Giordano, A., Bocci, E.B., Bistoni, O., Vaudo, G., et al. (2004) CD4 + CD28 - T Lymphocytes Contribute to Early Atherosclerotic Damage in Rheumatoid Arthritis Patients. Circulation, 109, 2744-2748. https://doi.org/10.1161/01.CIR.0000131450.66017.B3

[35] Creemers, E.E., Cleutjens, J.P., Smits, J.F. and Daemen, M.J. (2001) Matrix Metalloproteinase Inhibition after Myocardial Infarction: A New Approach to Prevent Heart Failure? Circulation Research, 89, 201-210. https://doi.org/10.1161/hh1501.094396

[36] Mohammed, F.F., Smookler, D.S. and Khokha, R. (2003) Metalloproteinases, Inflammation, and Rheumatoid Arthritis. Annals of the Rheumatic Diseases, 62, ii43-ii47. https://doi.org/10.1136/ard.62.suppl_2.ii43

[37] Sandoo, A., Kitas, G.D., Carroll, D. and Veldhuijzen van Zanten, J.J. (2012) The Role of Inflammation and Cardiovascular Disease Risk on Microvascular and Macrovascular Endothelial Function in Patients with Rheumatoid Arthritis: A Cross-Sectional and Longitudinal Study. Arthritis Research \& Therapy, 14, R117. https://doi.org/10.1186/ar3847

[38] Sandoo, A., Chanchlani, N., Hodson, J., Smith, J.P., Douglas, K.M. and Kitas, G.D. (2013) Classical Cardiovascular Disease Risk Factors Associate with Vascular Function and Morphology in Rheumatoid Arthritis: A Six-Year Prospective Study. Arthritis Research \& Therapy, 15, R203. https://doi.org/10.1186/ar4396

[39] Giles, J.T., Szklo, M., Post, W., Petri, M., Blumenthal, R.S., Lam, G., et al. (2009) Coronary Arterial Calcification in Rheumatoid Arthritis: Comparison with the Multi-Ethnic Study of Atherosclerosis. Arthritis Research \& Therapy, 11, R36. https://doi.org/10.1186/ar2641

[40] Crowson, C.S., Rollefstad, S., Ikdahl, E., Kitas, G.D., van Riel, P.L.C.M., Gabriel, S.E., et al. (2018) A Trans-Atlantic Cardiovascular Consortium for Rheumatoid Arthritis (ATACC-RA). Impact of Risk Factors Associated with Cardiovascular Outcomes in Patients with Rheumatoid Arthritis. Annals of the Rheumatic Diseases, 77, 48-54. https://doi.org/10.1136/annrheumdis-2017-211735

[41] Ambrosino, P., Lupoli, R., Di Minno, A., Tasso, M., Peluso, R. and Di Minno, M.N. (2015) Subclinical Atherosclerosis in Patients with Rheumatoid Arthritis. A Meta-Analysis of Literature Studies. Thrombosis and Haemostasis, 113, 916-930. https://doi.org/10.1160/TH14-11-0921

[42] Kimhi, O., Caspi, D., Bornstein, N.M., Maharshak, N., Gur, A., Arbel, Y., et al. (2007) Prevalence and Risk Factors of Atherosclerosis in Patients with Psoriatic 
Arthritis. Seminars in Arthritis and Rheumatism, 36, 203-209. https://doi.org/10.1016/j.semarthrit.2006.09.001

[43] Shaharyar, S., Warraich, H., McEvoy, J.W., Oni, E., Ali, S.S., Karim, A.J., et al. (2014) Subclinical Cardiovascular Disease in Plaque Psoriasis: Association or Causal Link? Atherosclerosis, 232, 72-78. https://doi.org/10.1016/j.atherosclerosis.2013.10.023

[44] Ramonda, R., Lo Nigro, A., Modesti, V., Nalotto, L., Musacchio, E., Iaccarino, L., et al. (2011) Atherosclerosis in Psoriatic Arthritis. Autoimmunity Reviews, 10, 773-778. https://doi.org/10.1016/j.autrev.2011.05.022

[45] Enany, B., El Zohiery, A.K., Elhilaly, R. and Badr, T. (2012) Carotid Intima-Media Thickness and Serum Leptin in Psoriasis. Herz, 37, 527-533. https://doi.org/10.1007/s00059-011-3547-Z

[46] Troitzsch, P., Paulista Markus, M.R., Dörr, M., Felix, S.B., Jünger, M., Schminke, U., et al. (2012) Psoriasis Is Associated with Increased Intima-Media Thickness-The Study of Health in Pomerania (SHIP). Atherosclerosis, 225, 486-490. https://doi.org/10.1016/j.atherosclerosis.2012.09.026

[47] Balci, D.D., Balci, A., Karazincir, S., Ucar, E., Iyigun, U., Yalcin, F., et al. (2009) Increased Carotid Artery Intima-Media Thickness and Impaired Endothelial Function in Psoriasis. Journal of the European Academy of Dermatology and Venereology, 23, 1-6. https://doi.org/10.1111/j.1468-3083.2008.02936.x

[48] Martyn-Simmons, C.L., Ranawaka, R.R., Chowienczyk, P., Crook, M.A., Marber, M.S., Smith, C.H., et al. (2011) A Prospective Case-Controlled Cohort Study of Endothelial Function in Patients with Moderate to Severe Psoriasis. British Journal of Dermatology, 164, 26-32. https://doi.org/10.1111/j.1365-2133.2010.10031.x

[49] Zardi, E.M. and Afeltra, A. (2010) Endothelial Dysfunction and Vascular Stiffness in Systemic Lupus Erythematosus: Are They Early Markers of Subclinical Atherosclerosis? Autoimmunity Reviews, 9, 684-686. https://doi.org/10.1016/j.autrev.2010.05.018

[50] Kiss, E., Soltesz, P., Der, H., Kocsis, Z., Tarr, T., Bhattoa, H., et al. (2006) Reduced Flow-Mediated Vasodilation as a Marker for Cardiovascular Complications in Lupus Patients. Journal of Autoimmunity, 27, 211-217. https://doi.org/10.1016/j.jaut.2006.09.008

[51] Sinicato, N.A., da Silva Cardoso, P.A. and Appenzeller, S. (2013) Risk Factors in Cardiovascular Disease in Systemic Lupus Erythematosus. Current Cardiology Reviews, 9, 15-19. https://doi.org/10.2174/157340313805076304

[52] Margiotta, D.P.E., Basta, F., Dolcini, G., Batani, V., Navarini, L. and Afeltra, A. (2017) The Relation between, Metabolic Syndrome and Quality of Life in Patients with Systemic Lupus Erythematosus. PLoS ONE, 12, e0187645.

https://doi.org/10.1371/journal.pone.0187645

[53] Gustafsson, J.T. and Svenungsson, E. (2014) Definitions of and Contributions to Cardiovascular Disease in Systemic Lupus Erythematosus. Autoimmun, 47, 67-76. https://doi.org/10.3109/08916934.2013.856005

[54] De Leeuw, K., Freire, B., Smith, A.J., Bootsma, H., Kallenberg, C.G. and Bijl, M. (2006) Traditional and Non-Traditional Risk Factors Contribute to the Development of Accelerated Atherosclerosis in Patients with Systemic Lupus Erythematosus. Lupus, 15, 675-682. https://doi.org/10.1177/0961203306069972

[55] Wu, G.C., Liu, H.R., Leng, R.X., Li, X.P., Li, X.M., Pan, H.F., et al. (2016) Subclinical Atherosclerosis in Patients with Systemic Lupus Erythematosus: A Systemic Review and Meta-Analysis. Autoimmunity Reviews, 15, 22-37. 
https://doi.org/10.1016/j.autrev.2015.10.002

[56] Salmon, J.E. and Roman, M.J. (2001) Accelerated Atherosclerosis in Systemic Lupus Erythematosus: Implications for Patient Management. Current Opinion in Rheumatology, 13, 341-344. https://doi.org/10.1097/00002281-200109000-00001

[57] Gistera, A. and Hansson, G.K. (2017) The Immunology of Atherosclerosis. Nature Reviews Nephrology, 13, 368-380. https://doi.org/10.1038/nrneph.2017.51

[58] Soltész, P., Dér, H., Kerekes, G., Szodoray, P., Szücs, G., Dankó, K., et al. (2009) A Comparative Study of Arterial Stiffness, Flow-Mediated Vasodilation of the Brachial Artery, and the Thickness of the Carotid Artery Intima-Media in Patients with Systemic Autoimmune Diseases. Clinical Rheumatology, 28, 655-662. https://doi.org/10.1007/s10067-009-1118-y

[59] Sacre, K., Escoubet, B., Pasquet, B., Chauveheid, M.P., Zennaro, M.C., Tubach, F., et al. (2014) Increased Arterial Stiffness in Systemic Lupus Erythematosus (SLE) Patients at Low Risk for Cardiovascular Disease: A Cross-Sectional Controlled Study. PLoS ONE, 9, e94511. https://doi.org/10.1371/journal.pone.0094511

[60] Valero-Gonzalez, S., Castejon, R., Jimenez-Ortiz, C., Rosado, S., Tutor-Ureta, P., Vargas, J.A., et al. (2014) Increased Arterial Stiffness Is Independently Associated with Metabolic Syndrome and Damage Index in Systemic Lupus Erythematosus Patients. Scandinavian Journal of Rheumatology, 43, 54-58.

https://doi.org/10.3109/03009742.2013.803150

[61] Selzer, F., Sutton-Tyrrell, K., Fitzgerald, S., Tracy, R., Kuller, L. and Manzi, S. (2001) Vascular Stiffness in Women with Systemic Lupus Erythematosus. Hypertension, 37, 1075-1082. https://doi.org/10.1161/01.HYP.37.4.1075

[62] Trojanowska, M. (2010) Cellular and Molecular Aspects of Vascular Dysfunction in Systemic Sclerosis. Nature Reviews Rheumatology, 6, 453-460. https://doi.org/10.1038/nrrheum.2010.102

[63] Manetti, M., Guiducci, S., Romano, E., Rosa, I., Ceccarelli, C., Mello, T., et al. (2013) Differential Expression of Junctional Adhesion Molecules in Different Stages of Systemic Sclerosis. Arthritis \& Rheumatology, 65, 247-257. https://doi.org/10.1002/art.37712

[64] Sfikakis, P.P., Tesar, J., Baraf, H., Lipnick, R., Klipple, G. and Tsokos, G.C. (1993) Circulating Intercellular Adhesion Molecule-1 in Patients with Systemic Sclerosis. Clinical Immunology and Immunopathology, 68, 88-92. https://doi.org/10.1006/clin.1993.1100

[65] Szamosi, S., Csiki, Z., Szomják, E., Szolnoki, E., Szoke, G., Szekanecz, Z., et al. (2009) Plasma Homocysteine Levels, the Prevalence of Methylenetetrahydrofolate Reductase Gene C677T Polymorphism and Macrovascular Disorders in Systemic Sclerosis: Risk Factors for Accelerated Macrovascular Damage? Clinical Reviews in Allergy \& Immunology, 36, 145-149. https://doi.org/10.1007/s12016-008-8105-y

[66] Ihn, H., Fujimoto, M., Sato, S., Kikuchi, K., Igarashi, A., Soma, Y., et al. (1994) Increased Levels of Circulating Intercellular Adhesion Molecule-1 in Patients with Localized Scleroderma. Journal of the American Academy of Dermatology, 31, 591-595. https://doi.org/10.1016/S0190-9622(94)70221-7

[67] Kiener, H., Graninger, W., Machold, K., Aringer, M. and Graninger, W.B. (1994) Increased Levels of Circulating Intercellular Adhesion Molecule-1 in Patients with Systemic Sclerosis. Clinical and Experimental Rheumatology, 12, 483-487.

[68] Beckman, J.S. and Koppenol, W.H. (1996) Nitric Oxide, Superoxide, and Peroxynitrite: The Good, the Bad, and Ugly. American Journal of Physiology, 271, C1424-C1437. https://doi.org/10.1152/ajpcell.1996.271.5.C1424 
[69] Szucs, G., Tímár, O., Szekanecz, Z., Dér, H., Kerekes, G., Szamosi, S., et al. (2007) Endothelial Dysfunction Precedes Atherosclerosis in Systemic Sclerosis-Relevance for Prevention of Vascular Complications. Rheumatology (Oxford), 46, 759-762. https://doi.org/10.1093/rheumatology/kel426 